\title{
Structure antigénique du Nématode Angiostrongylus cantonensis.
}

Aspects immunologiques des relations hôte-parasite.

\author{
par F. Bouthemy, A. CAPRon, D. AFCHAIn et P. WATtré \\ Service d'Immunologie et de Biologie parasitaire, Centre hospitalier et universitaire de Lille, \\ place de Verdun, $F 59000$ Lille
}

\begin{abstract}
Résumé
Angiostrongylus cantonensis, parasite du rat et agent de la méningite à éosinophiles de l'homme, présente une mosaïque antigénique complexe. Parmi les 25 composants antigéniques mis en évidence par l'analyse immuno-électrophorétique de l'extrait de vers adultes, deux semblent jouer un rôle prépondérant. L'étude de l'extrait antigénique larvaire n'a permis de révéler que quinze composants. Il existe des parentés antigéniques restreintes avec les Cestodes et les Trématodes (2) et plus nombreuses avec les Nématodes (6). Des parentés ont été observées entre le parasite, l'hôte intermédiaire et l'hôte définitif et peuvent expliquer un des aspects des modalités de l'adaptation parasitaire.

La connaissance théorique de l'antigène Angiostrongylus cantonensis a été complétée par l'étude des anticorps précitants élaborés au cours de l'angiostrongylose expérimentale du rat en fonction de la chronologie et de l'intensité de l'infestation.

Chez l'homme où Angiostrongylus cantonensis est en impasse parasitaire et ne dépasse pas le stade larvaire, les anticorps précipitants sont mis en évidence aussi bien par l'antigène extrait d'adultes que de larves.
\end{abstract}

\section{Summary}

The antigenic structures of Angiostrongylus cantonensis, Nematode parasite of the rat, responsible for human eosinophilic meningitis, has been studied.

Among the 25 antigenic components identified by immunoelectrophoretic analysis, in the adult worm extract, two appear 
to be particularly immunogenic. 15 components were demonstrated in an extract of the larval form. Cross reactions are weak with antigenic extracts of Cestodes and Trematodes (2) but more important with antigens from Nematodes (6). Common antigens have been observed between $A$. cantonensis and the intermediate host $B$. glabrata as well as with the definitive host and may explain some aspects of parasite adaptation.

Precipitating antibodies, during the experimental infection of the rat, were studied according to the age of infection and the number of parasites.

In human disease where $A$. cantonensis do not develop after the larval stage precipitating antibodies may be demonstrated with adult extract as well as larval extract.

\section{Introduction}

Au cours de recherches sur les parasites animaux des rats de Canton, H. T. Chen (1935) [9] mit en évidence des nématodes filiformes qu'il décrivit sous le nom de Pulmonema cantonensis, sans doute, à cause de leur localisation pulmonaire. Ce n'est qu'en 1945 que ce parasite fut redécouvert et redécrit par Nomura et Lin [17] sous le nom d'Angiostrongylus cantonensis. Les formes immatures, mises en évidence dans le liquide céphalo-rachidien d'un sujet de 15 ans atteint de méningite à éosinophiles ont pu être rattachées alors à l'espèce antérieurement décrite. Un intérêt tout particulier devait s'attacher à la description de ce parasite lorsque Alicata (1961) [2] découvrit qu'Angiostrongylus cantonensis était l'agent de la méningite à éosinophiles de l'homme à Tahiti.

Les nombreux travaux consacrés à Angiostrongylus cantonensis ont été analysés par Alicata (1965) [3], (1970) [4]. Ils concernent l'étude parasitologique, biologique, épidémiologique, clinique et diagnostique de ce parasite. Ils ne révèlent aucun élément précis, concernant d'une part la structure antigénique de ce parasite et d'autre part les réactions immunologiques qu'il est susceptible de déterminer tant chez l'hôte définitif (rat) que chez l'homme.

Nous nous sommes proposé, au cours de ce travail, d'étudier la structure antigénique d'Angiostrongylus cantonensis et les aspects immunologiques des relations hôte-parasite dans l'angiostrongylose humaine et expérimentale.

\section{Matériel et méthodes}

\section{I. - Le cycle expérimental d'Angiostrongylus cantonensis}

A) INFEstation DE L'Hôte DÉFINITIF.

Les méthodes d'infestation sont empruntées dans leurs grandes lignes au travail de Courdurier et coll. (1964) [11], (1966) [10], (1967) [12], (1968) [13], et modifiées en vue d'une production massive d'antigène. 
Des exemplaires de Biomphalaria glabrata *, infestés depuis vingt-huit jours au moins, sont privés de leur coquille, comprimés entre deux lames porte-objet, afin de contrôler à l'aide d'une loupe binoculaire, la présence de larves (L III) d'Angiostrongylus cantonensis. Le degré d'infestation est globalement apprécié, et un fragment est donné par voie buccale à chaque rat. La dose infectieuse optimale est d'environ cent larves par animal. Ce dernier développe, vingt-cinq à trente jours après l'infestation, un syndrome neurologique fait de convulsions accompagnées d'hémorragies nasales, qui va entraîner la mort dans 20 à $25 \%$ des cas. Les parasites s'installent dans les vaisseaux pulmonaires des rats survivants, et on peut retrouver des larves dans les excréments à partir du quarante-huitième jour. Les rats sont sacrifiés à cette époque, et la dissection des vaisseaux pulmonaires permettra la récolte des vers adutes. Ils serviviront à la préparation de l'antigène vermineux adulte.

L'obtention des larves (L IV) destinées à la préparation de l'antigène correspondant peut être réalisée en infestant massivement les rats et en les sacrifiant vingt-cinq à trente jours après l'infestation. Les larves sont aisément recueillies dans les espaces sous-arachnoïdiens.

\section{B) INFESTATION DE L'HÔTE INTERMÉDIAIRE.}

Des déjections de rats infestées de larves (L. I) du parasite sont délitées avec un peu d'eau dans une boîte de Pétri. Des groupes de dix mollusques sont mis au contact de ces déjections pendant sept à huit heures. Soigneusement et délicatement nettoyés ensuite avec un pinceau de soie, ils sont placés dans un aquarium convenablement aéré et éclairé, maintenu à une température de $23^{\circ} \mathrm{C}$.

Leur nourriture se compose de feuilles de salade et de poudre nutritive composée. Après avoir subi deux mues au bout de vingt-huit jours, les larves parviennent au stade (L III) et sont capables d'infester l'hôte définitif.

\section{II. - Matériel et techniques immunologiques}

\section{A) Antigènes.}

Les antigènes utilisés au cours de ce travail furent constitués par des extraits réalisés par broyage mécanique réfrigéré dans une solution de $\mathrm{NaCl} 0,017 \mathrm{M}$ de vers parasites récoltés vivants, lavés, broyés et centrifugés. Le surnageant est dialysé contre de l'eau distillée, et lyophilisé. Cet extrait constitue l'antigène somatique. L'antigène délipidé est obtenu par utilisation d'éther rendu anhydre, rectifié et distillé au moment de l'emploi.

On a préparé ainsi des extraits antigéniques de vers adultes et de «larves L IV » recueillis dans les espaces sous-arachnoïdiens.

\% Nous devons cette souche à l'amabilité du Docteur Richards (Laboratory of Parasitic diseases, National Institute of Allergy and infectious diseases, National Institute of Health, Bethesda, U.S.A.) qui a eu l'obligeance de nous confier quelques exemplaires de Biomphalaria glabrata infestés. 


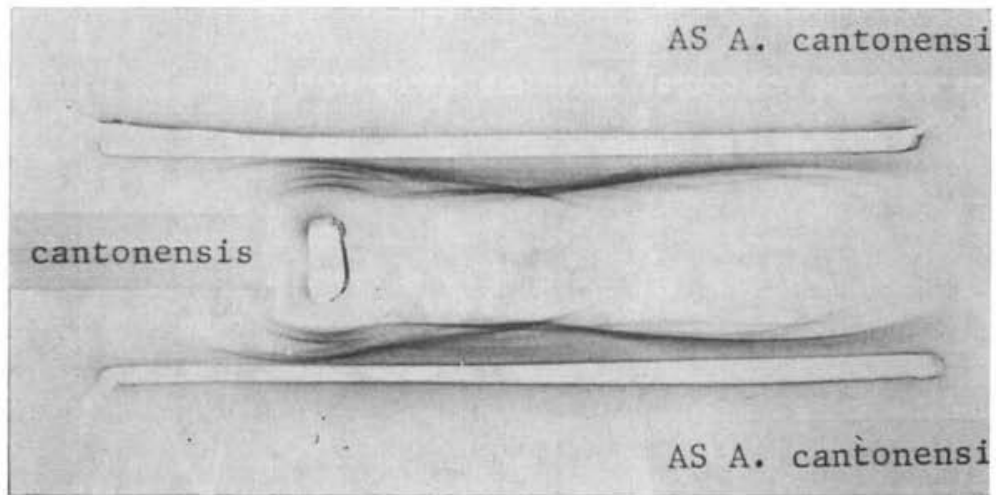

Рното А. - Analyse immuno-électrophorétique des antigènes de Angiostrongylus cantonensis (lapin hyperimmunisé)

B) SÉrums.

Trois types de sérums furent utilisés :

- des sérums de lapins hyperimmunisés par une injection sous-cutanée hebdomadaire de $20 \mathrm{mg}$ d'extrait antigénique additionné d'adjuvant de Freund incomplet ;

- des sérums de rats infestés suivant le protocole précédemment décrit ;

- 16 échantillons de sérums de malades atteints d'angiostrongylose *.

\section{C) Techniques d'immunodiffusion.}

La méthode d'Ouchterlony (1948) [19] et sa variante selon Abelev, (1960) [1] furent utilisées pour évaluer le rapport des concentrations antigène-anticorps le plus favorable à l'immuno-électrophorèse et préciser l'existence des réactions croisées et l'identification partielle ou totale de certaines fractions antigéniques.

L'analyse immuno-électrophorétique selon Grabar et Williams, (1953) [14], (1955) [15] fut appliquée en suivant le protocole opératoire décrit dans de précédents travaux [5].

Après électrophorèse préalable réalisée en gel d'agarose durant $5 \mathrm{~h} 30$ sous un potentiel de $20 \mathrm{~V}$ (tampon véronal sodé : $\mathrm{pH} 8,2$; f.i. 0,1 ) aux extrémités des lames, les fractions antigéniques sont révélées par les immunsérums concentrés trois fois; les arcs de précipitation sont colorés à l'amidoschwarz, et les activités enzymatiques sont caractérisées selon les techniques préconisées par Uriel et coll. et appliquées aux antigènes parasitaires (1961) [20], (1963) [21].

* Nous devons ces sérums à l'extrême obligeance du Médecin-Colonel Saugrain (Directeur de 1'I.R.M.L.M., Papete, Tahiti) à qui nous exprimons nos très vifs remerciements. Nous remercions également le Docteur Loison (Directeur du Programme Santé, Commission du Pacific Sud, Nouméa Nouvelle-Calédonie) pour l'aide si bienveillante et le rôle décisif qu'il a joué dans l'acheminement de cet intéressant matériel. 


\section{Résultats}

\section{Structure antigénique.}

\section{I. - Mosaïque antigénique}

A. - L'analyse immuno-électrophorétique des antigènes de « vers adultes » Angiostrongylus cantonensis a permis de révéler 25 fractions antigéniques, numérotées arbitrairement de 1 à 25 de la cathode à l'anode. Ces fractions correspondent aux 25 arcs de précipitation qui ont été mis en évidence quand on oppose à cet extrait antigénique un sérum de lapin hyperimmunisé (schéma 1, photo A).

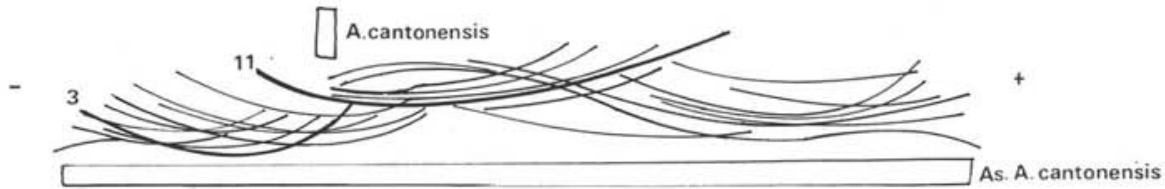

SchÉma 1. - Analyse des antigènes du ver adulte Angiostrongylus cantonensis révélés après électrophorèse par un immunsérum de lapin homologue

L'existence possible de contaminants d'origine sérique ou tissulaire de l'hôte, nous a amenés à saturer le sérum de lapin précédemment étudié avec des antigènes sériques ou tissulaires de rat. Après cette absorption, 4 des 25 fractions ont disparu (schéma 2).

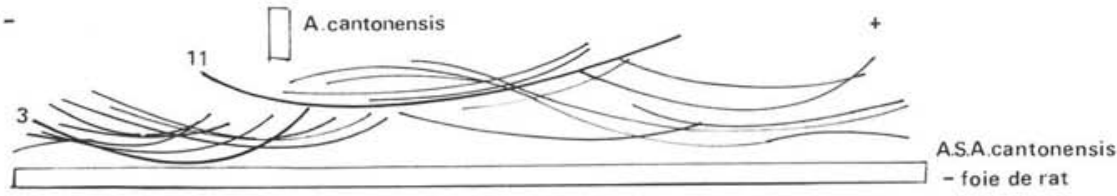

SchÉma 2. - Analyse des antigènes du ver adulte Angiostrongylus cantonensis révélés après électrophorèse par un immunsérum de lapin homo'ogue après absorption par les antigènes foie de rat

Cette complexité se retrouve au niveau des antigènes larvaires. Cependant, le nombre de systèmes précipitants révélés en immuno-électrophorèse par un immunsérum antiadultes n'atteint jamais un taux aussi élevé qu'avec les antigènes des « vers adultes »; ceux-ci ne comportent que 15 fractions antigéniques (schéma 3 ).

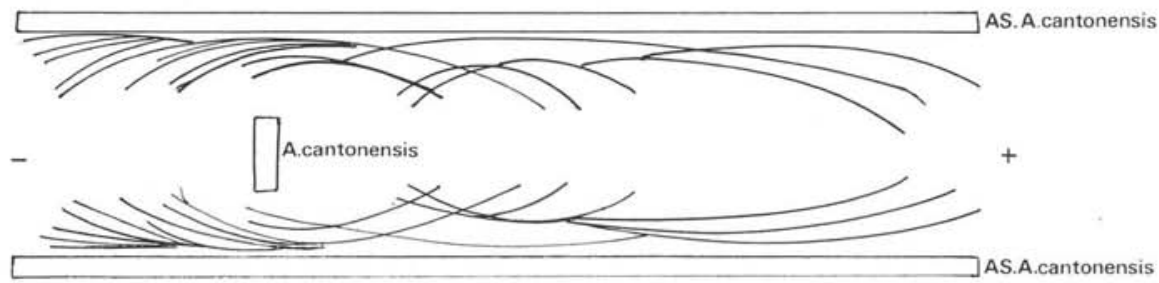

SCHÉmA 3. - Analyse des antigènes de la larve du cerveau Angiostrongylus cantonensis révélés après électrophorèse par un immunsérum de lapin homologue 
B. - L'immunogénicité variable de chacune des fractions antigéniques a été considérée :

- d'une part en étudiant l'ordre d'apparition des anticorps précipitants élaborés au cours de l'immunisation expérimentale du lapin;

- d'autre part en appréciant l'intensité plus ou moins marquée des différents arcs de précipitation.

a) ORDRE D'APPARITION DES ARCS.

Dès la fin de la première semaine de l'immunisation des lapins, apparaissent deux arcs de précipitation sur les immuno-électrophorégrammes, et six à la deuxième semaine ; à cette ascension rapide du nombre des systèmes précipitants succède une production plus lente et plus régulière, jusqu'à la mise en évidence de seize systèmes précipitants à la onzième semaine.

L'hyperimmunisation du lapin est constatée à la treizième semaine, où l'on retrouve sur l'immuno-électrophorégramme les 25 complexes antigène-anticorps fondamentaux (schéma 4).

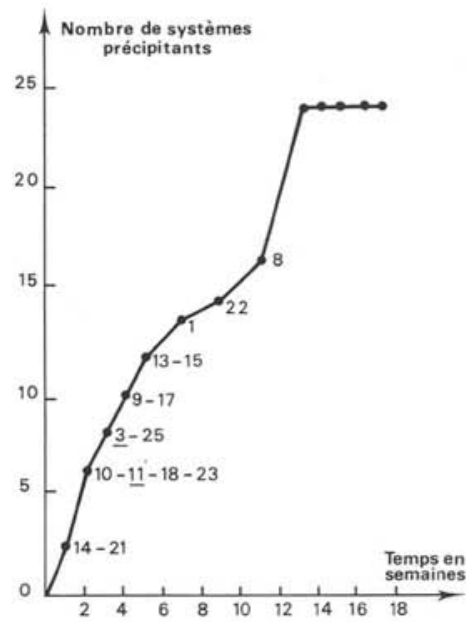

SCHÉma 4. - Courbe évolutive de l'apparition et de l'identification des systèmes précipitants chez le lapin hyperimmunisź par un extrait soluble d'Angiostrongylus cantonensis.

\section{b) INTENSITÉ DES ARCS.}

Parmi les 25 arcs de précipitation, 2 d'entre eux, plus marqués dans leur intensité, n'apparaissent pas intéressés dans les réactions croisées avec d'autres Helminthes, et sont présents lorsque l'hyperimmunsérum a été absorbé sur de l'antigène foie de rat.

Ces deux composants antigéniques d'apparition très rapide, sont donc très immunogènes ; celui qui apparaît le premier, dès la deuxième semaine, est le onzième à partir du pôle négatif ; l'autre, de mobilité électrophorétique plus cathodique, apparaît dès la troisième semaine et correspond à l'arc de précipitation $\mathrm{n}^{\circ} 3$. 


\section{II. - Parentés antigéniques avec les Helminthes}

Les travaux de Biguet et coll. (1965) [5] avaient mis l'accent sur l'homogénéité relative de structure des Nématodes.

Capron et coll. (1968) [6] étudièrent les parentés antigéniques de 11 espèces de Trématodes, 12 espèces de Cestodes et 12 espèces de Nématodes, parasites de l'homme ou des animaux domestiques. Ils furent amenés à envisager certains aspects immunologiques des relations hôte-parasite chez les Helminthes et à émettre de nouvelles hypothèses concernant le processus général d'adaptation parasitaire. Nous avons noté l'importance des parentés antigéniques qui unissent Angiostrongylus cantonensis et divers Nématodes (Tableau I).

\section{Tableau I}

PaRentés antigéniques UNISSANT Angiostrongylus cantonensis a dES NÉMATOdes

TABLEAU $1 a$

Parentés avec des filiaires

\begin{tabular}{|c|c|c|c|c|}
\hline Antisérums & $\begin{array}{c}\text { Dipetalonema } \\
\text { viteae }\end{array}$ & $\begin{array}{c}\text { Dirofilaria } \\
\text { immitis }\end{array}$ & $\begin{array}{c}\text { Onchocerca } \\
\text { volvulus }\end{array}$ & $\begin{array}{c}\text { Litomosoïdes } \\
\text { carinii }\end{array}$ \\
\cline { 2 - 4 } $\begin{array}{c}\text { Antigène } \\
\text { A. cantonensis }\end{array}$ & 7 & 5 & 6 & 3 \\
\hline
\end{tabular}

TABLEAU $1 b$

Parentés avec des Ascaris.

\begin{tabular}{|c|c|c|c|c|}
\hline Antisérums & $\begin{array}{c}\text { Ascaris } \\
\text { lumbricoires }\end{array}$ & $\begin{array}{c}\text { Toxocara } \\
\text { canis }\end{array}$ & $\begin{array}{c}\text { Ascaris } \\
\text { suum } \\
\text { (Liquide } \\
\text { equorum } \\
\text { coelomique) }\end{array}$ \\
\hline $\begin{array}{c}\text { Antigène } \\
\text { cantonensis }\end{array}$ & 7 & $\begin{array}{l}8 \text { (adultes) } \\
\text { (larves) }\end{array}$ & 5 & 4 \\
\hline
\end{tabular}

TABLEAU $1 c$

Parentés avec des Nématodes libres.

\begin{tabular}{|c|c|c|c|}
\hline \hline Antisérums & $\begin{array}{c}\text { Caenorhabditis } \\
\text { elegans }\end{array}$ & $\begin{array}{c}\text { Rhabditis } \\
\text { sp. }\end{array}$ & $\begin{array}{c}\text { Panagrellus } \\
\text { silusiae }\end{array}$ \\
\hline $\begin{array}{c}\text { Antigène } \\
\text { cantonensis }\end{array}$ & 4 & 3 & 5 \\
\hline \hline
\end{tabular}


Il existe en moyenne six fractions communes avec les Filaroidea et six avec les Ascaridoidea.

Dipetalonema viteae. Ascaris lumbricoides. Toxocara canis présentent sept fractions communes avec Angiostrongylus cantonensis. Mais l'examen des immunoélectrophorégrammes montre qu'une seule fraction se retrouve dans les trois diagrammes. Il est particulièrement intéressant de noter qu'Angiostrongylus cantonensis possède avec les Nématodes libres Rhabdita (Rhabditis sp., Caenorabditis elegans, Panagrellus silusiae) quatre fractions communes, ce qui confirme les observations faites par Capron et coll. (1968) [6] sur l'homogénéité de structure des Nématodes et sur l'existence d'une structure phylétique originelle.

Ces observations sont en accord avec les hypothèses de Chabaud (1965) [8j (schéma 5) sur la filiation des différents types de cycles évolutifs chez certains Phasmidiens, la systématique des Nématodes reposant sur une phylogénie qui correspond en réalité à une adaptation de plus en plus parfaite au parasitisme.

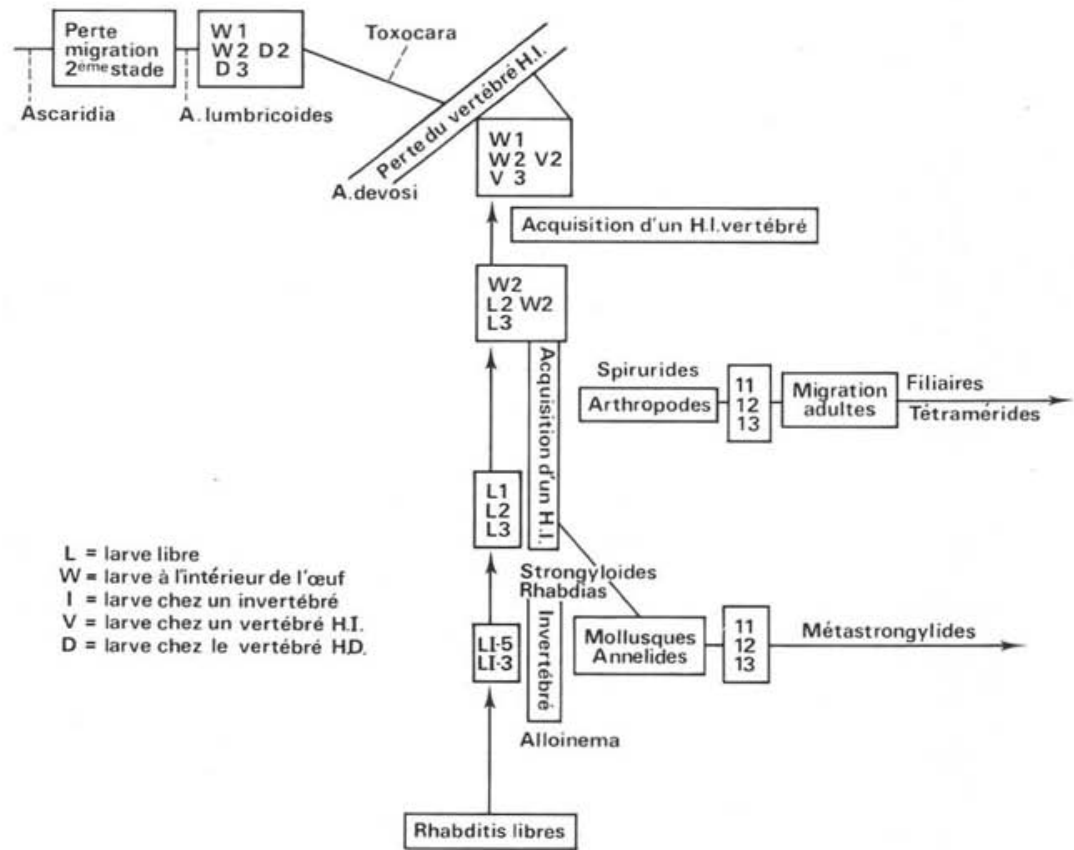

Schéma 5. - Hypothèse sur la filiation des différents types de cycles évolutifs chez certains Phasmidiens selon Chabaud (schéma simplifié)

Les Métastrongylides représentent la première étape de la vie hétéroxène.

Les hôtes intermédiaires (Mollusques, Annélides) ont une séclusion * faible, et dans certains cas le développement larvaire correspond presque à un ectoparasitisme.

* Séclusion: Réactions indirectes par lesquelles les organismes tendent à se soustraire aux influences du milieu extérieur. 
Les Strongylida, en effet, gardent des affinités manifestes avec les formes libres. La structure larvaire en particulier reste proche de celle des Rhabditis, et pour les adultes, Osche (1958) [18] a pu établir la filiation entre les papilles cloacales des Rhabditis et les côtes bursales des Strongylida qui ne sont que des papilles modifiées.

Les espèces qui sont à l'origine d'un phylum possèdent une mosaïque de protéines «primitives». Au cours de l'évolution, les espèces nouvelles perdent certaines de ces protéines initiales pour les remplacer par des nouvelles qui constituent sans doute les composants caractéristiques des espèces, tandis que ceux, communs à l'ensemble des espèces, peuvent être représentatifs du phylum des Nématodes.

Le remaniement structural des espèces chez les Nématodes parasites a été d'autant plus indispensable que leurs ancêtres étaient libres. Il a donc fallu, lors de l'adaptation à la vie parasitaire, un modelage des mécanismes biochimiques et immunochimiques en fonction de ceux de leurs hôtes.

Toutefois, la part de la structure protéique phylétique ou «mosaïque originelle * a été peu modifiée, et chez les Nématodes les communautés antigéniques sont nombreuses.

Il est à noter enfin que les communautés entre Angiostrongylus cantonensis et les Cestodes (Tableau II) d'une part, et les Trématodes (Tableau III) d'autre part, sont moins importantes et ne se révèlent guère que par l'existence de deux fractions de faible intensité sur les immuno-électrophorégrammes.

\section{Tableau II}

Parentés antigéniques unissant Angiostrongylus cantonensis à des Cestodes.

\begin{tabular}{|c|c|c|c|}
\hline \hline Antisérums & $\begin{array}{c}\text { Taenia } \\
\text { saginata }\end{array}$ & $\begin{array}{c}\text { Echinococcus } \\
\text { granulosus } \\
\text { (liquide hydatique) }\end{array}$ & $\begin{array}{c}\text { Taenia } \\
\text { solium }\end{array}$ \\
\cline { 2 - 4 } $\begin{array}{c}\text { Antigène } \\
\text { cantonensis }\end{array}$ & 2 & 3 & 1 \\
\hline
\end{tabular}

\section{Tableau III}

Parentés antigéniques unissant Angiostrongylus cantonensis à des Trématodes.

\begin{tabular}{|c|c|c|c|}
\hline \hline Antisérums & $\begin{array}{c}\text { Fasciola } \\
\text { hepatica }\end{array}$ & $\begin{array}{c}\text { Schistosoma } \\
\text { mansoni }\end{array}$ & $\begin{array}{c}\text { Paragonimus } \\
\text { westermani }\end{array}$ \\
\cline { 2 - 4 } $\begin{array}{c}\text { Antigène } \\
\text { cantonensis }\end{array}$ & 0 & $\begin{array}{l}3 \text { (adultes) } \\
5 \text { (larves) }\end{array}$ & 0 \\
\hline
\end{tabular}




\section{III. - Parentés antigéniques hôte-parasite}

Les relations unissant le parasite à son hôte définitif et à son hôte intermédiaire ont été considérées.

L'hôte définitif d'Angiostrongylus cantonensis est un rongeur du genre rattus. Aussi ont été étudiées par des méthodes immuno-électrophorétiques, non seulement les parentés antigéniques qui pouvaient exister entre ce parasite et le rat, mais aussi avec d'autres rongeurs.

Les antigènes utilisés pour ces études ont été constitués par des sérums et des extraits solubles du foie de ces rongeurs.

Un extrait soluble de poumon de rat fut également utilisé comme antigène à cause de la localisation pulmonaire des vers adultes. Les résultats obtenus sont rassemblés dans le tableau IV.

\section{Tableau IV}

Communautés antigéniques unissant Angiostrongylus cantonensis à certains rongeurs.

\begin{tabular}{|c|c|c|c|c|c|c|c|c|c|}
\hline \multirow{2}{*}{ Antigènes } & \multicolumn{2}{|c|}{ Souris } & \multicolumn{3}{|c|}{ Rat } & \multicolumn{2}{|c|}{ Hamster } & \multicolumn{2}{|c|}{ Mérion } \\
\hline & Foie & Sérum & Foie & Sérum & $\begin{array}{l}\text { Pou- } \\
\text { mon }\end{array}$ & Foie & Sérum & Foie & Sérum \\
\hline $\begin{array}{l}\text { Antisérum } \\
\text { A. canto- } \\
\text { nensis ... }\end{array}$ & 1 & 2 & 6 & 6 & 6 & 1 & 3 & 3 & 2 \\
\hline
\end{tabular}

Il existe six déterminants antigéniques communs entre Angiostrongylus cantonensis et le rat. Les réactions d'Ouchterlony montrent que le déterminant antigénique commun entre Angiostrongylus et le foie de souris est le même que celui qui existe entre Angiostrongylus et le foie de hamster. De même, l'identité immunologique est démontrée pour trois des fractions communes qui existent entre Angiostrongylus et le sérum, le foie, ou le poumon du rat.

Les fractions antigéniques communes entre Angiostrongylus et l'hôte intermédiaire utilisé, Biomphalaria glabrata, sont au nombre de trois.

Ces observations apparaissent comparables à celles de Capron et coll. (1968) concernant Schistosoma mansoni. Angiostrongylus cantonensis et Schistosoma mansoni sont tous deux des parasites qui, expérimentalement, ont été étudiés avec Biomphalaria glabrata comme hôte intermédiaire et avec un rongeur comme hôte définitif. 


\section{Tableau V}

Analyse comparée des antigènes $S$. mansoni et $A$. cantonensis par rapport aux hôtes intermédiaires et définitifs

\begin{tabular}{|c|c|c|}
\hline & $\begin{array}{l}\text { Hôte intermédiaire } \\
\mathrm{Nb} \text {. de systèmes précipitants }\end{array}$ & $\begin{array}{c}\text { Hôte définitif } \\
\mathrm{Nb} \text {. de systèmes précipitants }\end{array}$ \\
\hline Schistosoma mansoni & 6 & .6 \\
\hline $\begin{array}{l}\text { Angiostrongylus } \\
\text { cantonensis } \quad \ldots \ldots \ldots\end{array}$ & 3 & $\begin{array}{l}\text { foie } \\
\text { sérum } \\
\text { poumon }\end{array}$ \\
\hline
\end{tabular}

Si l'on compare les antigènes « vers adultes » et les antigènes « larvaires 》 d'Angiostrongylus cantonensis, on constate qu'ils n'ont pas la même valeur antigénique.

\section{Tableau VI}

Analyse comparée des antigènes « vers adultes » et «larves du cerveau » d'Angiostrongylus cantonensis

\begin{tabular}{|c|c|c|}
\hline & $\begin{array}{c}\text { Antigène adulte: } \\
\text { Nb. de systèmes précipitants }\end{array}$ & $\begin{array}{l}\text { Antigène larvaire: } \\
\mathrm{Nb} \text {. de systèmes précipitants }\end{array}$ \\
\hline Sérum de rat sain. & 4 & 2 \\
\hline Foie de rat sain ... & 6 & 6 \\
\hline B. glabrata ...... & 3 & 3 \\
\hline $\begin{array}{l}\text { Sérum de rat infesté } \\
\text { depuis } 72 \text { jours } \\
\text { (nombre maximum) }\end{array}$ & 12 & 6 \\
\hline
\end{tabular}

Le troisième point de ce tableau, qui retient l'attention, est le fait qu'un sérum de rat infesté depuis 72 jours, donc bien longtemps après la maturité sexuelle des vers, n'ait pas la même réponse immunologique vis-à-vis des antigènes «vers adultes » et « larves cérébrales ».

\section{IV. - Caractérisation des activités enzymatiques}

L'analyse immunoélectrophorétique de la structure antigénique d'Angiostrongylus cantonensis, l'étude des communautés antigéniques que ce ver présente avec d'autres helminthes ou avec les tissus des hôtes intermédiaires et définitifs ont été complétées par la recherche de la signification physiologique éventuelle de certains composants antigéniques révélés. 
Un essai de mise en évidence des différents types d'activités enzymatiques, supportées par les complexes antigène-anticorps des immuno-électrophorégrammes des broyats d'Angiostrongylus cantonensis, révélés par les hyperimmunsérums expérimentaux homologues, a été fait en utilisant les méthodes en milieu gélifié préconisées par Uriel (1961) [20], (1963) [21].

L'analyse immuno-électrophorétique des antigènes Angiostrongylus cantonensis fournie par un hyperimmunsérum homologue de lapin a permis de révéler 7 types différents d'activités enzymatiques supportées par 11 des 25 complexes antigène-anticorps révélés (schéma 6).

$$
\begin{aligned}
& \mathrm{L}=\text { lactico-déhydrogénase. } \\
& \mathrm{MD}=\text { malico-déhydrogénase. } \\
& \mathrm{A}=\mathrm{L} \text {. Alanine-déhydrogénase. } \\
& \beta=6 \text { hydroxy butyro-déhydrogénase. } \\
& \mathrm{G}=\text { glutamo-déhydrogénase. } \\
& \mathrm{C}=\text { catalase. } \\
& \mathrm{E}=\text { estérase carboxylique. }
\end{aligned}
$$

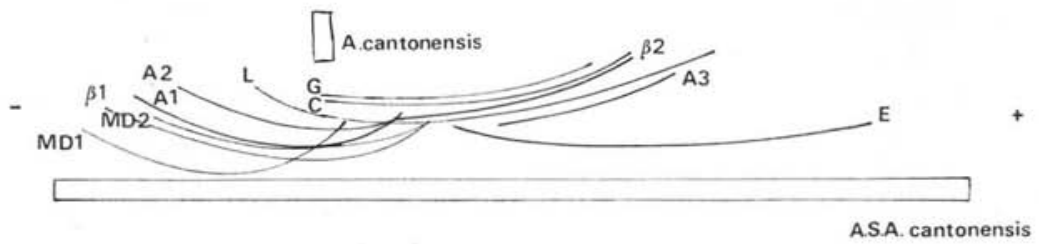

Schéma 6. - Activités enzymatiques objectivées lors de l'analyse des antigènes du ver adulte Angiostrongylus cantonensis révélés après électrophorèse par un immunsérum de lapin homologue

Les activités enzymatiques sont surtout des oxydo-réductions, c'est-à-dire des hydrogènes transférases ou déshydrogénases.

- Cinq types d'activités déshydrogénasiques utilisant le nicotinamide adénine dinucléotide (N.A.D.) ou le nicotinamide adénine dinucléotide phosphate (N.A.D.P.) comme transporteur d'hydrogène, ont été mis en évidence. Notons que l'identification précise du type de la déshydrogénase observée peut être sujet à critique ; en effet, des interférences de réaction peuvent être observées entre la L-alanine déshydrogénase et la lactico déshydrogénase, par exemple, et il n'est pas possible de conclure de façon spécifique quant à la détermination exacte du type d'activité déshydrogénasique en cause.

- Un type d'activité deshydrogénasique utilisant $\mathrm{H}_{2} \mathrm{O}_{2}$ comme accepteur a également été mis en évidence, il s'agit d'une catalase. lique.

- Parmi les hydrolases, seule a pu être mise en évidence une estérase carboxy- 


\section{Anticorps sériques \\ dans l'angiostrongylose expérimentale du rat}

La structure antigénique d'Angiostrongylus cantonensis a été complétée par une étude des anticorps sériques dans l'angiostrongylose expérimentale du rat (photo B).

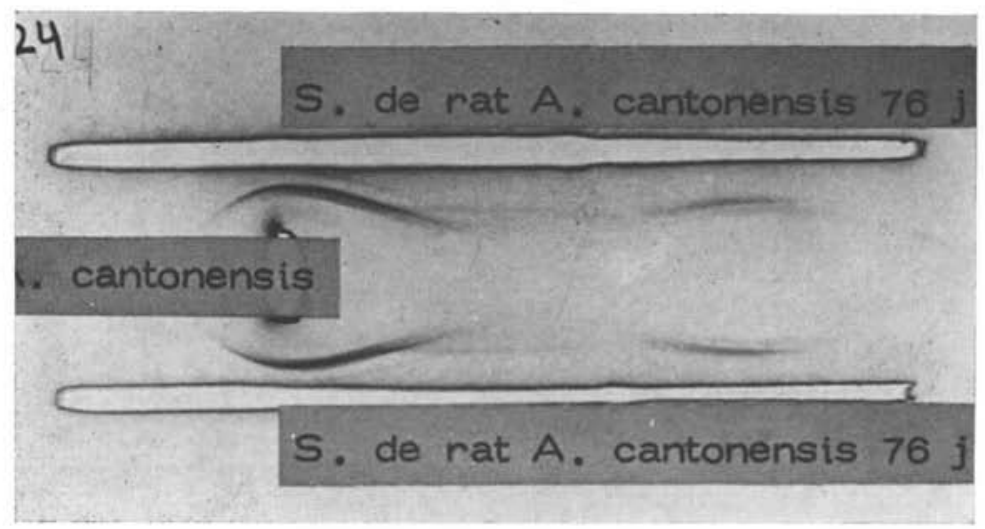

Рното В. - Anticorps précipitants décelés au cours de l'Angiostrongylose expérimentale du Rat, 76 jours après l'infestation

\section{Evolution chronologique des anticorps précipitants}

Dans ce but, plusieurs centaines de rats furent infestés par les méthodes précédemment décrites. Les sérums furent étudiés par les techniques d'immunodiffusion classiques, mais avec un extrait antigénique Angiostrongylus cantonensis délipidé.

La courbe évolutive de l'apparition des systèmes précipitants chez le rat (schéma 7) peut se décomposer en plusieurs parties :

- La première, qui correspond à une période de 1 à 45 jours, est caractérisée par une augmentation, d'abord très rapide jusqu'au $10^{\circ}$ jour, puis plus progressive jusqu'au $45^{\circ}$ jour, du nombre des anticorps précipitants. La production importante d'anticorps précipitants observés sur les immuno-électrophorégrammes ( 3 arcs de précipitation) pendant les 10 premiers jours suivant l'infestation du rat est probablement due à l'introduction chez celui-ci des larves infestantes provenant des mollusques.

Ensuite, une stimulation métabolique due à la maturation des vers Angiostrongylus cantonensis engendre une réponse plus atténuée, mais cependant importante.

- La seconde partie de la courbe, du $45^{\circ}$ au $70^{\circ}$ jour, indique une brève diminution du nombre des anticorps précipitants, suivie d'une remontée.

La diminution transitoire, du $45^{\circ}$ au $60^{\circ}$ jour, du taux des anticorps précipitants trouve peut-être son explication dans la maturité sexuelle des vers et la ponte d'œufs 
par les femelles. Ce phénomène avait été observé lors de la distomatose hépatique par Capron (1965) [7].

Ce lâcher d'antigène va provoquer une nouvelle réaction de l'organisme qui va se traduire par une production massive d'anticorps du $60^{\circ}$ au $70^{\circ}$ jour.

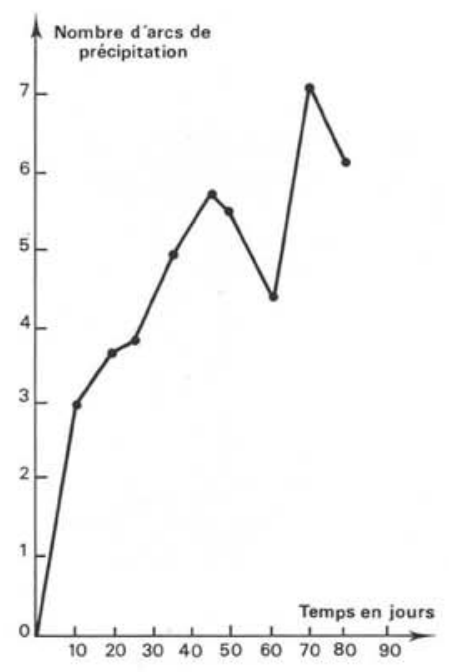

Schéma 7. - Courbe évolutive de l'apparition des systèmes précipitants chez le rat expérimentalement infecté par Angiostrongylus cantonensis

- La dernière partie de la courbe qui, dans les limites de notre expérimentation, s'achève au $90^{\circ}$ jour, amorce une décroissance qui peut être motivée par une diminution du nombre des vers.

Il faut également noter que Courdurier et coll. (1964) [11] ont obtenu une courbe d'allure généralement semblable à la nôtre, lors de l'étude de l'éosinophilie sanguine chez le rat infesté expérimentalement par Angiostrongylus cantonensis. L'hyperéosinophilie est caractérisée par deux phases: la première atteignant son maximum au $6^{\circ}$ jour au moment où les larves ont atteint le cerveau et la seconde atteignant un taux nettement plus élevé au $46^{\circ}$ jour, lorsque le parasite achève sa maturité sexuelle.

\section{Evolution des anticorps précipitants en fonction du degré d'infestation}

La dose d'infestation par larves des rats étant pratiquement impossible à évaluer, la courbe de l'apparition des systèmes précipitants a été faite en fonction du nombre des vers adultes retirés des poumons lors de la dissection des rats. 
Cette courbe (schéma 8) présente une similitude avec celle établie et étudiée par Lavier (1944) [16], d'après les chiffres de Maass. Ce dernier avait exprimé la variation du taux des éosinophiles en fonction du nombre de trichines infestantes chez le porc. Il avait montré que l'éosinophilie parasitaire n'apparaît qu'avec une masse parasitaire minimale et qu'il y avait une certaine proportionnalité entre la quantité infestante et l'intensité de l'éosinophilie.

SCHÉma 8. - Courbe de l'apparition des systèmes précipitants en fonction du degré de l'infection chez le rat expérimentalement infecté depuis plus de 45 jours par Angiostrongy'us cantonensis

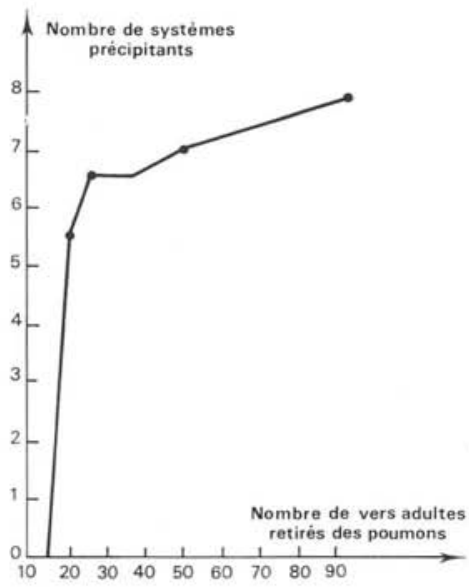

Dix vers adultes, dans les poumons, constituent la masse parasitaire minimale d'Angiostrongylus cantonensis pour entraîner l'apparition d'anticorps précipitants décelables par nos techniques.

Lorsque dix à vingt vers arrivent à maturité dans les poumons, la réponse immunologique est importante, puisqu'une moyenne théorique de cinq à six arcs est obtenue en immuno-électrophorèse. Plus l'infestation est massive, plus cette réponse s'intensifie. Par contre, elle n'augmente plus de façon significative au-delà de quatre-vingt vers adultes.

Il semble donc qu'il y ait, comme pour la courbe d'éosinophilie étudiée par Lavier, un phénomène de saturation immunologique.

Cependant, jamais plus de cent soixante vers n'ont été retirés des poumons de rats infectés; on ignore donc si, après cette phase de saturation, existe une phase d'inhibition comme l'a montré Shutsu Young cité par Lavier (1944) [16] en établissant la courbe d'éosinophilie en fonction des quantités infestantes, lors de l'infestation expérimentale du lapin par Clonorchis sinensis. 


\section{Anticorps sériques \\ dans l'angiostrongylose humaine}

L'analyse immuno-électrophorétique a été appliquée à seize sérums de malades atteints de méningite à éosinophiles en pays d'endémie et attribuables à Angiostrongylus cantonensis, ainsi qu'à des sérums de référence provenant de sujets sains ou atteints d'autres parasitoses.

Les résultats obtenus montrent la sensibilité et la spécificité de cette technique dans le diagnostic de l'angiostrongylose.

\section{Sensibilité de la réaction}

13 parmi les 16 sérums donnent lieu à une précipitation en gélose (1 à 4 arcs) avec un extrait antigénique «vers adultes».

Ce nombre est de 10 (1 à 3 arcs) vis-à-vis d'un extrait antigénique «larves du cerveau ».

L'état d'altération des sérums, en raison du long délai qu'ils ont mis à nous parvenir, explique peut-être les difficultés de lecture et le nombre relativement bas des précipitines. Les résultats auraient probablement été meilleurs avec des sérums plus frais.

Par ailleurs, il paraît surprenant d'observer quatre systèmes précipitants avec l'antigène «vers adultes » et trois seulement avec l'antigène «larves du cerveau », alors que le parasite n'atteint jamais le stade adulte chez l'homme.

\section{Spécificité de la réaction}

La spécificité de l'analyse immuno-électrophorétique est appréciée en opposant des sérums humains à différents antigènes de nématodes: D. viteae, T. canis, A. suum. Le tableau VII indique que le maximum d'arcs de précipitation obtenus sur les immuno-électrophorégrammes est de 2 pour 4 des 16 sérums opposés à un extrait antigénique $D$. viteae, pour 2 des 16 sérums opposés à un extrait antigénique $T$. canis, pour 1 des 16 sérums opposés à un extrait antigénique $A$. suum.

Si l'on tient compte des réactions croisées éventuelles et la possibilité des réactions aspécifiques, il est évident que le diagnostic de l'angiostrongylose par la précipitation en gélose sera d'autant moins sujet à erreur que les systèmes précipitants seront plus nombreux. La qualité des sérums a aussi une très grande importance. 


\section{Tableau VII}

Etude de la spécificité de l'analyse immunoélectrophorétique: analyse comparée des antigènes de divers Nématodes révélés après électrophorèse par des sérums de malades atteints de méningite à éosinophiles attribuée à Angiostrongylus cantonensis

\begin{tabular}{|c|c|c|c|c|c|c|}
\hline \multirow{3}{*}{$\begin{array}{l}\text { Sórum } \\
\text { humain }\end{array}$} & & \multicolumn{5}{|c|}{ Nombre de systèmes précipitants } \\
\hline & & \multirow[b]{2}{*}{ D. vitae } & \multirow[b]{2}{*}{ T. canis } & \multirow[b]{2}{*}{ A. suum } & \multicolumn{2}{|c|}{ A. cantonensis } \\
\hline & $\mathrm{dg}$ & & & & Ag. adulte & $\begin{array}{c}\text { Ag. lar- } \\
\text { vaire }\end{array}$ \\
\hline AND. & & & & & & \\
\hline $1^{\circ}$ prélèvement & $\ldots \ldots \ldots \ldots$ & Néant & Néant & Néant & Néant & Néant \\
\hline $2^{\circ}$ prélèvement & $\ldots \ldots \ldots \ldots$ & Néant & Néant & Néant & Néant & Néant \\
\hline GAD. & & & & & & \\
\hline $1^{\circ}$ prélèvement & $\ldots \ldots \ldots \ldots$ & 2 & 2 & 2 & 3 & 3 \\
\hline $2^{\circ}$ prélèvement & $\ldots \ldots \ldots$ & 2 & 1 & 1 & 3 & 3 \\
\hline ELL. & & & & & & \\
\hline $1^{\circ}$ prélèvement & $\ldots \ldots \ldots \ldots$ & Néant & Néant & Néant & 1 & 2 \\
\hline $2^{\circ}$ prélèvement & $\ldots \ldots \ldots \ldots$ & Néant & Néant & Néant & 1 & Néant \\
\hline HER. & & & & & & \\
\hline $1^{\circ}$ prélèvement & $\ldots \ldots \ldots \ldots$ & 1 & Néant & Néant & 1 & 1 \\
\hline $2^{\circ}$ prélèvement & $\ldots \ldots \ldots \ldots$ & 1 & 1 & 1 & 1 & Néant \\
\hline JAC. & & & & & & \\
\hline $1^{\circ}$ prélèvement & $\ldots \ldots \ldots \ldots$ & 1 & Néant & Néant & 2 & 2 \\
\hline $2^{\circ}$ prélèvement & $\ldots \ldots \ldots \ldots$ & 1 & Néant & 1 & 2 & 1 \\
\hline JAM. & & & & & & \\
\hline $1^{\circ}$ prélèvement & $\ldots \ldots$ & 2 & 2 & 1 & 3 & 3 \\
\hline $2^{\circ}$ prélèvement & $\ldots \ldots \ldots \ldots$ & 2 & 1 & 1 & 4 & 3 \\
\hline SPI. & & & & & & \\
\hline $1^{\circ}$ prélèvement & $\ldots \ldots \ldots \ldots$ & Néant & Néant & Néant & Néant & Néant \\
\hline $2^{\circ}$ prélèvement & $\ldots \ldots \ldots \ldots$ & Néant & Néant & Néant & 2 & 1 \\
\hline URI. & & & & & & \\
\hline $1^{\circ}$ prélèvement & $\ldots \ldots$ & 1 & Néant & Néant & 1 & 1 \\
\hline $2^{\circ}$ prélèvement & $\ldots \ldots \ldots \ldots$ & 1 & Néant & Néant & 1 & Néant \\
\hline
\end{tabular}

\section{Conclusion}

Il apparaît, au terme du présent travail, que les objectifs que nous nous étions fixés n'ont pu être atteints que d'une manière partielle.

Angiostrongylus cantonensis, parasite du rat et agent de la méningite à éosinophiles de l'homme, présente une mosaïque antigénique complexe : 25 composants antigé- 
niques s'individualisent par l'analyse immuno-électrophorétique de l'extrait de vers adultes opposé à un hyperimmunsérum de lapin homologue, et 15 seulement avec un extrait antigénique larvaire. L'intérêt de cette analyse, outre la connaissance théorique qu'elle nous apporte du potentiel antigénique du parasite, nous renseigne sur le rôle privilégié joué par les composants antigéniques 11 et 3, par leur importance quantitative et par la production précoce dès les deuxième et troisième semaines, des anticorps précipitants correspondants. Ces deux arcs de précipitation objectivables sur la plupart des immuno-électrophorégrammes supportent une activité enzymatique déshydrogénasique et ne semblent pas intéressés dans les réactions croisées avec d'autres helminthes.

L'étude des réactions croisées met en évidence des parentés antigéniques peu nombreuses avec les Cestodes et les Trématodes (de 1 à 3 ) et plus nombreuses avec les Nématodes (jusqu'à 8). Dans le cas particulier des Nématodes, les parentés antigéniques paraissent liées aux déterminants communs à l'ensemble des espèces représentatives du phylum, selon Chabaud, ces déterminants spécifiques étant liés au remaniement structural des espèces chez les Nématodes parasites issus d'un ancêtre libre du type Rhabditis. L'adaptation parasitaire a donc peu modifié la part de la structure protéique phylétique.

Un des aspects des modalités de l'adaptation parasitaire a été récemment expliqué par le processus de synthèse, par le parasite, de protéines de comportement antigénique identique à celui des protéines de l'hôte intermédiaire.

Nous avons pu démontrer la réalité de ce phénomène par la mise en évidence de trois composants antigéniques communs entre Angiostrongylus cantonensis, tant à l'état larvaire qu'adulte, et le mollusque hôte intermédiaire, Biomphalaria grabrata. Les composants antigéniques communs entre Angiostrongylus cantonensis adulte et l'hôte définitif qui l'héberge (le rat), au nombre de six, sont d'interprétation plus difficile, car il peut s'agir d'une souillure de protéines de l'hôte définitif contenues dans le tube digestif du ver et non pas de protéines somatiques du ver.

Les recherches que nous venons de résumer ayant conduit à une connaissance théorique des antigènes Angiostrongylus cantonensis, nous nous sommes ensuite attachés à l'étude des anticorps précipitants élaborés au cours de l'angiostrongylose expérimentale et humaine.

L'étude de l'évolution des anticorps précipitants au cours de l'angiostrongylose expérimentale du rat montre que l'infestation du rat engendre, dès la période d'invasion, une production importante d'anticorps précipitants. La stimulation métabolique due au passage des vers à l'état adulte engendre une réponse immunitaire plus accentuée. La maturité sexuelle des vers, le lâcher d'antigène dû à la ponte des œufs par les femelles et, d'une manière générale, le métabolisme accru des vers adultes vont provoquer, après une diminution transitoire, une production massive d'anticorps dont les douze systèmes précipitants mis en évidence dans le sérum d'un rat infesté depuis soixante-douze jours en sont les témoins. 
La détermination du nombre de systèmes précipitants, en fonction de l'intensité de l'infestation, a permis de déterminer les faits suivants:

- une masse parasitaire minimale de dix vers adultes est nécessaire pour provoquer l'apparition d'anticorps précipitants ;

- il y a une relation, mais non une proportionnalité directe, entre quantité infestante et intensité de la réponse immunologique.

Ces résultats sont similaires à ceux obtenus par Maass chez le porc, lors de l'étude de la variation du taux maximum d'éosinophile en fonction des quantités infestantes de trichine.

Chez l'homme atteint d'angiostrongylose, le nombre maximum de systèmes précipitants mis en évidence dans les antigènes vers adultes est de quatre et de trois seulement avec l'antigène «larves de cerveau ». On peut considérer qu'il s'agit d'une anomalie, puisque Angiostrongylus cantonensis est en impasse parasitaire chez l'homme où il ne dépasse pas l'état larvaire. Le meilleur antigène ne paraît donc pas être, dans le cadre de notre étude, l'antigène strictement homologue.

\section{Bibliographie}

1. Abelev (G. I.) et Zvetkov (U. S.), 1960. - Isclation of precipitating antibodies to specific antigens of the liver and mouse hepatoma. Vop. Onkol., 6, 57-62 (Rus.).

2. Alicata (J. E.), 1961. - Personal letter to Dr Leon Rosen, National institutes of Health, Bethesda, Md., dated April 5, 1961, concerning Angiostrongylus as a probable cause of eosinophilic meningitis in Tahiti.

3. Alicata (J. E.), 1965. - Biology and distribution of the rat lung-worm, Angiostrongylus cantonensis, and its relation to eosinophilic meningitis and others neurological disorders of man and animal. Advances in Parasitology, Daves, B. (Ed), London and New York, Academic. Press., 3, 223-248.

4. Alicata (J. E.) and Jindrak (K.), 1970. - Angiostrongylosis in the Pacific and South East Asia. American lectures series edited by Hamilton H. Anderson Md. Lahaina, Mani, Hawaii.

5. Biguet (J.), Rose (F.), Capron (A.), et Tran Van Ky (P.), 1965. - Contribution de l'analyse immunoélectrophorétique à la connaissance des antigènes vermineux. Incidences pratiques sur leur standardisation, leur purification et le diagnostic des Helminthiases par immunoélectrophorèse. Rev. Immunol., 29, 5-30.

6. Capron (A.), Biguet (J.), Vernes (A.) et Afchain (D.), 1968. - Structure antigénique des Helminthes. Aspects immunologiques des relations hôte-parasite. Path. Biol., 16, 121-138.

7. Capron (A.), Rose (G.), Luffau (G.), Biguet (J.) et Rose (F.), 1965. - Apport de la Distomatose expérimentale à la connaissance de la Distomatose humaine à Fasciola hepatica. Aspects immunologiques. Rev. Immunol., 29, 25-41.

8. Chabaud (A. G.), 1965. - Cycles évolutifs des nématodes parasites de vertébrés, Dans Grasse. Traité de Zoologie, 4, fasc. 2 (Némathelminthes). Masson Ed. 
9. CHEN (H.-T.), 1935. - Un nouveau nématode pulmonaire, Pulmonema cantonensis. n. g. ; n. sp. des rats de Canton. Ann. Parasitol., 13, 312-317.

10. Courdurier (J.), Delgado y Garnica (R.), Lopez-Fernandez (J. R.) et Guillon (J.-C.), 1966. - Etudes des variations de l'éosinophilie sanguine chez le rat infesté expérimentalement par Angiostrongylus cantonensis. Bull. Soc. Path. Exot., 59, 359-365.

11. Courdurier (J.), Guillon (J.-C.), Malarde (L.), Laigret (J.), Desmoulin (G.) et Schollhammer (G.), 1964. - Réalisation du cycle d'Angiostrongylus cantonensis au laboratoire. I. Observations sur le cycle de l'ana:omopathologie causée par ce parasite chez divers animaux de laboratoire. Bull. Soc. Path. Exot., 57, 1253-1262.

12. Courdurier (J.), Guillon (J.-C.) et Malarde (L.), 1967. - Réalisation du cycle d'Angiostrongylus cantonensis (Chen), au labora:oire. II. Utilisation d'Australia glabratus (Say) comme hôte-intermédiaire. Contribution à l'étude du mode d'infestation du mollusque par les larves du parasite. Aspects histologiques. Bull. Soc. Path. Exot., 60, 165-173.

13. Courdurier (J.), Guillon (J.-C.) et Malarde (L.), 1968. - Réalisation du cycle d'Angiostrongylus cantonensis (Chen), au laboratoire. III. Les lésions chroniques des poumons chez les rats infestés expérimentalement. Bull. Soc. Path. Exot., 61, 254-257.

14. Grabar (P.) et Williams (C.-A.), 1953. - Méthode permettant l'étude conjuguée des propriétés électrophorétiques et immunochimiques d'un mélange de protéines. Application au sérum sanguin. Biochim. et Biophys. Acta, 10, 193-194.

15. Grabar (P.) et Williams (C.-A.), 1955. - Méthode immunoélectrophorétique d'analyse de mélanges de substances antigéniques. Biochim. et Biophys., Acta, 17, 67-74.

16. Lavier, 1944-1945. - L'éosinophilie sanguine dans les Helminthiases. Sang, 16, 510-528.

17. Nomura (S.) et LiN (H.), 1945. - First clinical case of Haemostrongylus ratti. (Japanese test). T'ai-Wan No Ikai, 3, 589-592.

18. Osche (G.), 1958. - Beitrage zur morphologie, okologie und phylogenic der Ascaridoidea (Nematoda), parallelen in der evolution von parasit und wirt. Zeisch. $f$. Parasitol., Bd., 18, 479-572.

19. Ouchterlony (O.), 1949. - Antigen-antibody reactions in gels. Acta Path. Microbiol. Scand., 26, 507-515.

20. URIEL (J.), 1961. - Caractérisation des cholinestérases et d'autres estérases carboxyliques après électrophorèse et immunoélectrophorèse en gélose. Ann. Inst. Pasteur, $101,104-119$.

21. URIEL (J.), 1963. - Characterization of enzymes in specific immune-precipitates. Ann. N. Y. Acad. Sci., 103, 956-979. 